University of Wollongong

Research Online

Australian Institute for Innovative Materials -

Papers

Australian Institute for Innovative Materials

$1-1-2014$

Controllable morphology of flux avalanches in microstructured superconductors

\author{
M Motta \\ Universidade Federal de Sao Carlos \\ F Colauto \\ Universidade Federal de Sao Carlos \\ J I. Vestgarden \\ University of Oslo, j.i.vestgarden@fys.uio.no \\ Joachim Fritzsche \\ Chalmers University of Technology \\ M Timmermans \\ Katholieke Universiteit Leuven
}

See next page for additional authors

Follow this and additional works at: https://ro.uow.edu.au/aiimpapers

Part of the Engineering Commons, and the Physical Sciences and Mathematics Commons

Research Online is the open access institutional repository for the University of Wollongong. For further information contact the UOW Library: research-pubs@uow.edu.au 


\title{
Controllable morphology of flux avalanches in microstructured superconductors
}

\begin{abstract}
The morphology of abrupt bursts of magnetic flux into superconducting films with engineered periodic pinning centers (antidots) has been investigated. Guided flux avalanches of thermomagnetic origin develop a treelike structure, with the main trunk perpendicular to the borders of the sample, while secondary branches follow well-defined directions determined by the geometrical details of the underlying periodic pinning landscape. Strikingly, we demonstrate that in a superconductor with relatively weak random pinning the morphology of such flux avalanches can be fully controlled by proper combinations of lattice symmetry and antidot geometry. Moreover, the resulting flux patterns can be reproduced, to the finest details, by simulations based on a phenomenological thermomagnetic model. In turn, this model can be used to predict such complex structures and to estimate physical variables of more difficult experimental access, such as the local values of temperature and electric field.
\end{abstract}

\section{Keywords}

controllable, microstructured, morphology, flux, superconductors, avalanches

Disciplines

Engineering | Physical Sciences and Mathematics

\section{Publication Details}

Motta, M., Colauto, F., Vestgarden, J. I., Fritzsche, J., Timmermans, M., Cuppens, J., Attanasio, C., Cirillo, C., Moshchalkov, V. V., Van de Dondel, J., Johansen, T. H., Ortiz, W. A. \& Silhanek, A. V. (2014). Controllable morphology of flux avalanches in microstructured superconductors. Physical Review B: Condensed Matter and Materials Physics, 89 (13), 134508-1-134508-7.

\section{Authors}

M Motta, F Colauto, J I. Vestgarden, Joachim Fritzsche, M Timmermans, J Cuppens, C Attanasio, C Cirillo, V V. Moshchalkov, J Van de Vondel, Tom H. Johansen, W A. Ortiz, and A V. Silhanek 


\title{
Controllable morphology of flux avalanches in microstructured superconductors
}

\author{
M. Motta, ${ }^{1}$ F. Colauto, ${ }^{1}$ J. I. Vestgården, ${ }^{2}$ J. Fritzsche,${ }^{3}$ M. Timmermans,,${ }^{4}$ J. Cuppens,${ }^{4}$ C. Attanasio, ${ }^{5}$ C. Cirillo, ${ }^{5}$ \\ V. V. Moshchalkov, ${ }^{4}$ J. Van de Vondel, ${ }^{4}$ T. H. Johansen, ${ }^{2,6}$ W. A. Ortiz, ${ }^{1}$ and A. V. Silhanek ${ }^{7}$ \\ ${ }^{1}$ Departamento de Física, Universidade Federal de São Carlos, 13565-905 São Carlos, São Paulo, Brazil \\ ${ }^{2}$ Department of Physics, University of Oslo, POB 1048, Blindern, 0316 Oslo, Norway \\ ${ }^{3}$ Department of Applied Physics, Chalmers University of Technology, S-412 96 Göteborg, Sweden \\ ${ }^{4}$ Institute for Nanoscale Physics and Chemistry, Nanoscale Superconductivity and Magnetism Group, Katholieke Universiteit Leuven, \\ Celestijnenlaan 200D, B-3001 Leuven, Belgium \\ ${ }^{5}$ CNR-SPIN Salerno and Dipartimento di Fisica "E. R. Caianiello", Università degli Studi di Salerno, Fisciano (Sa) I-84084, Italy \\ ${ }^{6}$ Institute for Superconducting and Electronic Materials, University of Wollongong, Northfields Avenue, \\ Wollongong, New South Wales 2522, Australia \\ ${ }^{7}$ Département de Physique, Université de Liège, B-4000 Sart Tilman, Belgium
}

(Received 14 December 2013; revised manuscript received 30 March 2014; published 14 April 2014)

\begin{abstract}
The morphology of abrupt bursts of magnetic flux into superconducting films with engineered periodic pinning centers (antidots) has been investigated. Guided flux avalanches of thermomagnetic origin develop a treelike structure, with the main trunk perpendicular to the borders of the sample, while secondary branches follow welldefined directions determined by the geometrical details of the underlying periodic pinning landscape. Strikingly, we demonstrate that in a superconductor with relatively weak random pinning the morphology of such flux avalanches can be fully controlled by proper combinations of lattice symmetry and antidot geometry. Moreover, the resulting flux patterns can be reproduced, to the finest details, by simulations based on a phenomenological thermomagnetic model. In turn, this model can be used to predict such complex structures and to estimate physical variables of more difficult experimental access, such as the local values of temperature and electric field.
\end{abstract}

DOI: 10.1103/PhysRevB.89.134508

PACS number(s): 74.25.Ha, 68.60.Dv, 74.78.-w

\section{INTRODUCTION}

The breakdown of a dielectric medium, with its typical dendritic electrostatic discharge, and the permanent damage left by a fracture propagating through a piece of material constitute a pair of examples of complex physical systems decaying suddenly from a high-energy to a lower-energy metastable state. As such, these phenomena share several common points, including (i) an extremely fast propagation; (ii) branching expansion, typically with ramifications avoiding each other; and (iii) an inherent irreproducibility, with no preferential paths. Strikingly similar features can be observed when magnetic-flux avalanches of thermomagnetic origin invade a superconducting film. Such avalanches consist of an abrupt propagation of a superconductor-normal interface, leaving behind a trail of overheated material. Such an interface rushes deeper into the sample in an attempt to establish the lowestenergy state, for which flux distribution should be smooth throughout the system. The supersonic propagation of these fronts can exceed by several orders of magnitude the typical speed of individual flux quanta [1]. For this reason, one cannot expect to draw meaningful predictions about evolution and shape of avalanches by simply extrapolating the dynamics of individual quantized superconducting vortices.

Although the physics behind the propagation of cracks, dielectric breakdowns, and flux avalanches have been extensively studied in uniform media [2-7], little is known about the pattern formation in the case of periodic variations in the properties of the host material. In particular, one can then pose the question as to whether the morphology of flux avalanches is reflecting microscopic properties of the matrix or, inversely, if by introducing modulations on the material properties one would be able to impose a particular shape to the ubiquitous multibranching splitting of avalanches. It follows then that findings in the domain of flux avalanches will bring about implications to other complex systems with which this problem shares the above-discussed similarities.

Microstructured thin superconducting films are unrivaled toy-model systems to study avalanches, in view of their simplified two-dimensional structures, which facilitate the fabrication of the sample, and the fact that the experiment can be carried out repeatedly on the very same system without physical consequences to the material. Let us now have a closer look at the current understanding of these phenomena. In superconducting materials, the flow of electric currents is typically inhomogeneously distributed, being more concentrated at the borders, owing to the finite magnetic penetration depth. In the ideal case of a superconducting film with perfectly flat borders, vortices are able to nucleate and cross the surface barriers once the current density at the edge approaches the depairing current [8,9]. A different situation emerges if the current streamlines at the edge are forced to depart from their rectilinear trajectory, for instance in order to circumvent a nonsuperconducting object or follow the contour of the sample [10,11]. Under these circumstances, current streamlines conglomerate at the sharp bends they encounter on their path, thus leading to local magnifications of the current density $[12,13]$. This so-called current crowding effect, relevant when no vortices — or only a few of them-are present in microscopic samples, has been recently recognized [14] to cause, in corner shaped superconducting microstrips, an asymmetric vortex dynamics, allowing for preferential penetration from the inner concave angle of the strip. 
A similar asymmetry in the flux penetration in corner shaped samples is also present at the macroscopic level, as recently demonstrated using magneto-optical (MO) imaging [15]. In this limit, it is possible to accurately model the flux dynamics by nonlocal electrodynamics, appropriate for thin-film geometry, which has proven to reproduce, to the finest details, the experimental observations regarding all peculiarities of flux penetration. These simulations have also shown that the electric field is much more intense at the concave corner than anywhere else in the film, a sign of the occurrence of an intensive flux transport through that spot.

It is thus clear that concave corners in a superconducting film are preferential spots for flux penetration, irrespective of the sample size, both in the microscopic as well as in the macroscopic domain. In addition, since high electric fields are known to trigger thermomagnetic avalanches $[6,16,17]$, it is thus likely that thin superconductors with concave corners are far more susceptible to the occurrence of such dramatic events than specimens with convex corners or without corners at all. Notice that this concept applies equally to a superconductor with a square hole filled with flux: if the magnetic pressure pushes the flux trapped in the hole to invade the superconducting frame, it would thus have preferential directions, defined by the inner corners. In circular rings, in contrast, no preferential directions would exist.

In this work we demonstrate that the existence of such preferential directions is an essential ingredient defining the morphology of catastrophic flux avalanches in superconductors with periodic arrays of holes [antidots (ADs)]. In order to provide unambiguous evidence of these effects we fabricated thin films of $\mathrm{Nb}$ and $\mathrm{Mo}_{79} \mathrm{Ge}_{21}$ containing identical square arrays of holes, with the particularity that one half of the film is decorated with square holes, whereas the other half has circular holes. Currents circulating around rounded holes do not give rise to preferential directions for flux movement, and avalanches taking place in such systems should follow the symmetry of the antidot lattice, i.e., paths forming treelike structures with secondary branches at $90^{\circ}$ of the main trunk. In contrast to that, square holes in a square lattice induce the so-called Christmas Tree morphology, with secondary branches making angles of $45^{\circ}$ with the main trunk.

As a matter of fact, avalanches with secondary branches at $90^{\circ}$ of the main bole have been reported by Vlasko-Vlasov et al. [18], Menghini et al. [19], and Motta et al. [20]. The observations described in the first of those papers also include $45^{\circ}$ branching, a case which was treated theoretically by Aranson et al. [21]. In the existing literature, however, no attempt has been made to associate the form of those guided avalanches to the geometrical details of the underlying pinning landscape, i.e., lattice symmetry and AD geometry. This paper reveals the intimate relationship existing between those features, an account of which was so far missing in the literature.

Although anisotropic vortex distributions have also been reported in the smooth penetration regime of superconducting films with periodic arrays of antidots [22-24], the present results should not be seen as a straightforward extension of that work, since abrupt flux avalanches involve a propagating interface separating a superconducting region from a nonsuperconducting domain, which is locally heated above the critical temperature. In other words, a far more complex behavior than simple vortex hopping dynamics needs to be taken into account in the case of flux avalanches.

It is worth mentioning that, having chosen $\mathrm{Nb}$ and $\mathrm{Mo}_{79} \mathrm{Ge}_{21}$, which have superconducting Ginzburg-Landau parameters $\kappa$ differing by an order of magnitude, we were able to confirm that the resulting avalanche morphology is largely defined by geometrical aspects and not by materialdependent parameters. However, intrinsic pinning, abundant in polycrystalline $\mathrm{Nb}$ and very weak in amorphous $\mathrm{Mo}_{79} \mathrm{Ge}_{21}$, also plays a role in the avalanche morphology.

\section{SAMPLE DETAILS}

The samples were lithographically defined films using two different superconducting materials, namely, amorphous $\mathrm{Mo}_{79} \mathrm{Ge}_{21}$ deposited by pulsed laser deposition with thickness of $25 \mathrm{~nm}$ and $\mathrm{Nb}$ deposited by UHV dc magnetron sputtering with thickness of $50 \mathrm{~nm}$, both on top of $\mathrm{Si} / \mathrm{SiO}_{2}$ substrates. The characteristics of the patterned samples appear in Table I. In addition, a scheme of the lattice symmetry and AD geometry of the specimens is shown in each magneto-optical image in the Results. The samples MoGe-I, MoGe-II, Nb-I, and Nb-II have a square lattice symmetry ( $\square$ ), whereas the specimen MoGe-III presents a centered rectangular 2D Bravais lattice $(\square)$. This pattern is obtained by displacing every row by one-half of the repetition length in its own direction and by a full length in the orthogonal direction. The samples MoGe-I and Nb-I have antidots with circular shape $(\bigcirc$, diameter $=1.5 \mu \mathrm{m})$ on one half of the sample and square shape $(\square$, side $=1.5 \mu \mathrm{m})$ on the other half, as shown in Fig. 1; in both samples the antidots are displayed in a $4-\mu \mathrm{m}$ square lattice.

Using the temperature derivative of the upper critical field near $T_{c}$ and the dirty limit expressions [25], the zero-temperature superconducting coherence length $\xi(0)$ and penetration depth $\lambda(0)$ shown in Table I were determined for the $\mathrm{Nb}$ film. For MoGe samples, $\xi(0)$ was determined using the same strategy, whereas $\lambda(0)$ was obtained from transport measurements in a plain thin film, through use of Eq. (A13) of [26]. Two important features influencing the choice of these materials are (i) the substantial difference in pinning strength of the virgin (unpatterned) materials, being MoGe, the material with the weakest pinning, and (ii) the large difference in the Ginzburg-Landau $\kappa$ parameter, being the largest $\kappa \sim 100$ for MoGe.

TABLE I. Lattice symmetry and size, AD geometry and size, superconducting critical temperature $T_{c}$, coherence length $\xi(0)$, penetration depth $\lambda(0)$, and lateral dimensions $l$ and $w$ for the samples investigated.

\begin{tabular}{lccccccc}
\hline \hline Sample & $\begin{array}{c}\text { Lattice } \\
(\mu \mathrm{m})\end{array}$ & $\begin{array}{c}\mathrm{AD} \\
(\mu \mathrm{m})\end{array}$ & $\begin{array}{c}T_{c} \\
(\mathrm{~K})\end{array}$ & $\begin{array}{c}\xi(0) \\
(\mathrm{nm})\end{array}$ & $\begin{array}{c}\lambda(0) \\
(\mathrm{nm})\end{array}$ & $\begin{array}{c}l \\
(\mathrm{~mm})\end{array}$ & $\begin{array}{c}w \\
(\mathrm{~mm})\end{array}$ \\
\hline MoGe-I & $\square-4.0$ & $\square \bigcirc-1.5$ & 6.7 & 6 & $\sim 500$ & 1.0 & 1.0 \\
MoGe-II & $\square-1.5$ & $\square-0.4$ & 6.7 & 6 & $\sim 500$ & 2.6 & 2.0 \\
MoGe-III & $\square-3.0 / 6.0$ & $\square-2.0$ & 6.2 & 5 & $\sim 500$ & 1.0 & 1.0 \\
Nb-I & $\square-4.0$ & $\square \bigcirc-1.5$ & 8.3 & 12 & 92 & 2.5 & 2.5 \\
Nb-II & $\square-4.0$ & $\square-1.5$ & 6.8 & 8 & 132 & 5.0 & 5.0 \\
\hline \hline
\end{tabular}




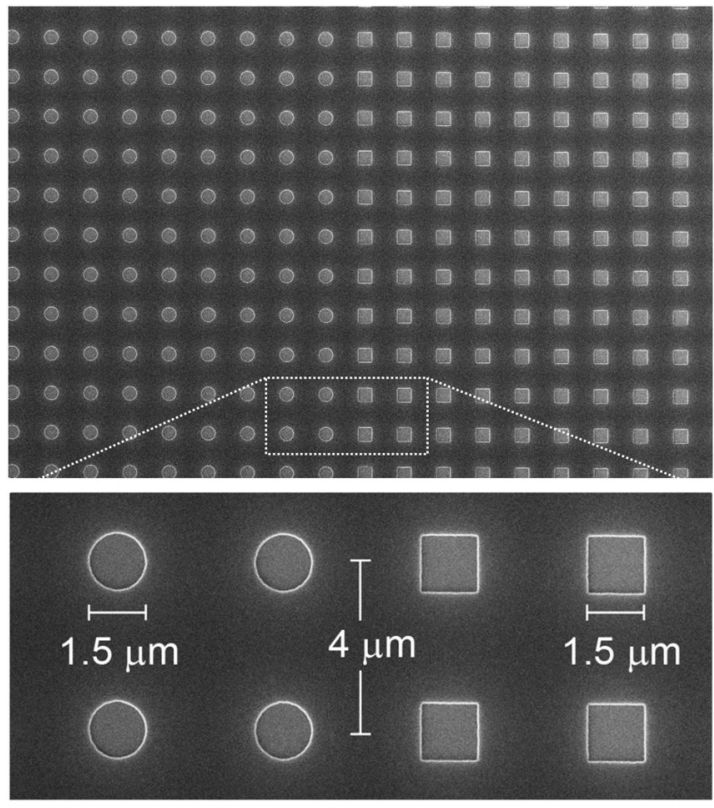

FIG. 1. Scanning electron microscopy image of the central portion of sample $\mathrm{Nb}-\mathrm{I}$, showing the abrupt change in antidot geometry, from circle (left) to square (right). Lattice parameter and AD sizes are shown in the zoomed up bottom panel.

The magneto-optical technique employed for imaging the flux penetration morphology is based on the occurrence of the Faraday effect in an indicator film placed on top of the superconducting specimen [27]. The indicators used in the present work are Bi-substituted yttrium iron garnet films (Bi:YIG) with in-plane magnetization [28]. As a consequence of its large $\lambda$, MoGe exhibits very weak magnetic-flux contrast and, to improve visual resolution, a zero-field background has been subtracted from all MO images involving MoGe. For $\mathrm{Nb}$ samples, for which the contrast is substantially larger, the subtraction procedure is not needed and raw images are shown.

\section{RESULTS AND DISCUSSIONS}

\section{A. Magneto-optical imaging}

Figure 2(a) presents a MO image of the magnetic flux penetrated in sample MoGe-I when an external magnetic field $H=1.2$ Oe was applied perpendicular to the sample surface after the sample was cooled down to $T=4.5 \mathrm{~K}$ (zero-field cooling process). Regions where magnetic flux is absent appear as black, whereas maximum field corresponds to the brightest intensity. The white rim along the perimeter of the sample indicates the high concentration of magnetic flux, a consequence of the large demagnetization factor in the perpendicular geometry.

A main feature of the image is the pair of dark diagonal lines extending across the sample. This additional shielding originates from the change in the direction of current flow when it adapts to the square shape of the film. These dark lines are at all four corners forming 45-deg angles with the sample edges, confirming that each half of the square behaves on a large scale as an isotropic film. Interestingly, also another feature is visible in the image, namely, dark lines extending into the film from the upper and lower edges from their respective midpoints. The line connecting these midpoints is the boundary between the two AD structures, and it is clear that the current does not flow straight across the boundary, i.e., the critical current density is not the same in the two halves of the sample.

Superimposed on the image in Fig. 2(a) is a drawing of the streamline pattern of the critical current flow, as suggested by the magneto-optical result. In addition to the 90-deg turns at the diagonals, sharp turns occur also at the boundary between the two $\mathrm{AD}$ structures. The streamline reconstruction is made by drawing sets of equidistant lines in each half of the square and then combining the two at the boundary [29]. The distance between the streamlines is inversely proportional to the respective critical current densities, $j_{\bigcirc}$ and $j_{\square}$, and it follows then that the angle $\beta$, seen in the figure, is related to the critical currents by

$$
j_{\square} / j_{\bigcirc}=-\cos 2 \beta .
$$
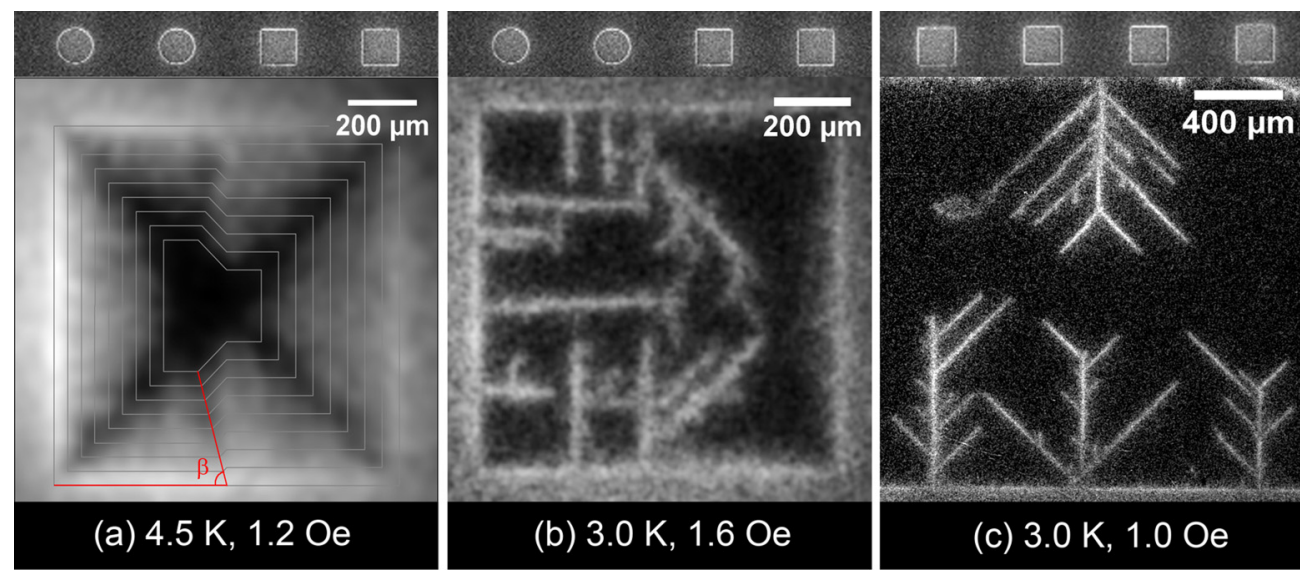

FIG. 2. (Color online) MO images for sample MoGe-I, with circular (left side) and square (right side) ADs, taken at (a) $T=4.5 \mathrm{~K}$ and $H=1.2 \mathrm{Oe}$, showing anisotropic flux penetration, and (b) $T=3 \mathrm{~K}$ and $H=1.6 \mathrm{Oe}$, revealing two different morphologies, depending on the AD geometry. (c) MO image for sample MoGe-II (square lattice of square ADs) taken at $T=3 \mathrm{~K}$ and $H=1$ Oe, showing avalanches with the Christmas Tree morphology. In panel (a) a drawing of the streamline pattern of the critical current flow is superimposed to the actual MO image. 

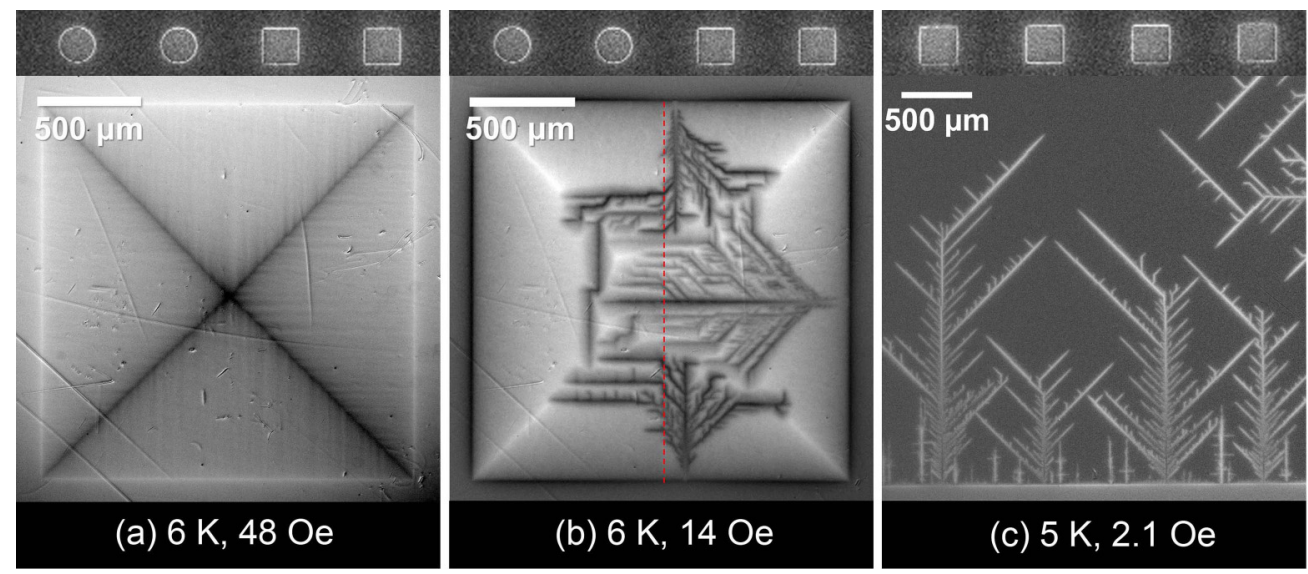

FIG. 3. (Color online) MO images taken for sample Nb-I, with circular (left side) and square (right side) ADs, taken at (a) $T=6 \mathrm{~K}$ and $H=48 \mathrm{Oe}$, showing isotropic flux penetration, and (b) $T=6 \mathrm{~K}$, for $H=14$ Oe (after decreasing from $H=48$ Oe), revealing different morphologies depending on the AD geometry. (c) MO image for sample Nb-II (square lattice of square ADs) taken at $T=5 \mathrm{~K}$ and $H=2.1$ Oe, showing avalanches with the Christmas Tree morphology.

Measuring from the image that $\beta=76^{\circ}$, we find that $j \square=$ $0.89 j_{\supset}$. A larger value for $j_{\bigcirc}$, as compared to $j_{\square}$, is fully consistent with the fact that the flux front entering from the right (square ADs) in Fig. 2(a) penetrates somewhat deeper than the corresponding front coming from the left (circular ADs). As a matter of fact round holes pin vortices more efficiently, even though such larger pinning strength cannot be attributed to the size of the hole, which is smaller for the circles. In reality, the difference is due to the sharp bends of currents around the square holes, which makes it easier for a vortex to escape the hole, leading to an effectively weaker pinning force.

At lower temperatures a dramatically different phenomenology takes place, since flux diffusion becomes faster, leaving not enough time for the local heat generated by vortex motion to be removed. Under these circumstances, magnetic-flux bursts that may achieve supersonic velocities invade the sample, typically forming a dendritic pattern very much like electrical discharges in a dielectric media. These thermomagnetic-flux avalanches, as shown in Fig. 2(b), can only develop if the slope of the flux gradient profile is steep enough or, in other words, if the critical current density is large. The fact that in Fig. 2(b) avalanches are triggered only from the side with rounded holes is consistent with the above-discussed feature that the critical current density for the part of the sample with this type of holes is larger than for the part with square holes.

It has been already shown that, due to the periodicity of the lattice of ADs, magnetic flux can be channeled mainly along the [10] and the [01] directions of the lattice [30,31]. This is also true for thermomagnetic avalanches, where fingerlike penetration consisting of a main trunk and small branches at $90^{\circ}$ has been observed in $\mathrm{Pb}$ and $\mathrm{Nb}$ samples with square arrays of antidots [19,20]. Figure 2(b) reveals the role of the AD geometry on the path followed by the invading flux: avalanches generated on the left side of the sample, showing a $90^{\circ}$ branching, transform themselves into $45^{\circ}$ branched tracks as soon as they cross the border and invade the right part of the film, which is patterned with square holes. This demonstrates that $90^{\circ}$ branching is a signature of flux avalanches propagating in an environment with a square array of round ADs, while the $45^{\circ}$ branching is the fingerprint of the underlying square array of square ADs. For comparison, Fig. 2(c) shows the formation of $45^{\circ}$ branching all across sample MoGe-II, which is entirely decorated with a square array of square holes. It is worth noting that in previous reports the emphasis was systematically put on the symmetry of the lattice, while the geometry of the holes was virtually ignored.

The question now arises as to whether the different morphology of the flux avalanches observed in sample MoGe-I for the square and rounded holes is material dependent. In order to explore this possibility we studied sample Nb-I, patterned with a hybrid array of circular and square holes, identical to the one introduced in sample MoGe-I. Since unpatterned $\mathrm{Nb}$ exhibits already a very strong pinning, we expect that drilling holes will not be as influential as in MoGe. This is indeed confirmed by the fact that the discontinuity lines [32] nearly follow the diagonals of the square sample, as shown in Fig. 3(a), thus indicating that the critical current density is not determined by the trapping of vortices in the holes. As a matter of fact, randomly distributed intrinsic pinning centers play a much more important role in $\mathrm{Nb}$ than in MoGe, to such an extent that reproducing, with sample Nb-I, the type of experiment depicted in Fig. 2(b) does not lead to the same clear-cut results in terms of morphology definition. However, in sample Nb-I, by first increasing the field up to a value large enough to warrant that the film is full of flux, and then decreasing it to a smaller value [see Fig. 3(b)], we observe $45^{\circ}$ branching, triggered from the right side of the film, which transforms itself into $90^{\circ}$ branching as soon as the line separating squares from circles is crossed. As a crosscheck, Fig. 3(c) presents avalanches occurring in sample Nb-II, entirely decorated with a square array of square antidots. Accordingly with Fig. 2(c), taken for sample MoGe-II, the Christmas Tree morphology is the only one appearing across the whole film [33].

\section{B. Modeling}

Once triggered, avalanches in superconducting films advance at very high speeds, typically ranging from units to several tens of $\mathrm{km} / \mathrm{s}[1,34,35]$, usually faster at the early stages. Such events are thus so fast that no instrumentation 
is currently available to capture a substantial set of images of such events while they occur. A viable alternative to obtain information about the spatial and temporal evolution of the dendritic flux avalanches is to carry out simulations of their dynamics. The origin of dendritic avalanches in superconducting films is a thermomagnetic instability mechanism due to the Joule heating created by vortex motion and the consequent reduction of the critical current density as the temperature increases [5,36]. The instability is also a consequence of the nonlinear material characteristics of type II superconductors, which is conventionally approximated by a power law:

$$
\mathbf{E}=\frac{\rho_{0}}{d}\left(\frac{J}{J_{c}}\right)^{n-1} \mathbf{J},
$$

where $\mathbf{E}$ is the electric field, $\mathbf{J}$ is the sheet current, $J=|\mathbf{J}|$, $\rho_{0}$ is a resistivity constant, $d$ is the sample thickness, $J_{c}$ is the critical sheet current, and $n$ is the creep exponent. The temperature dependencies are taken as

$$
J_{c}=J_{c 0}\left(1-T / T_{c}\right), \quad n=n_{0} T_{c} / T,
$$

where $T_{c}$ is the critical temperature. The electrodynamics must be supplemented by the heat diffusion equation:

$$
c \dot{T}=K \nabla^{2} T-\frac{h}{d}\left(T-T_{0}\right)+\frac{1}{d} J E,
$$

where $c$ is the specific heat, $K$ is the thermal conductivity, $h$ is the coefficient for heat removal to the substrate, and $T_{0}$ is the substrate temperature. The last term in Eq. (3) is the Joule heating, which provides the actual coupling between heat diffusion and electrodynamics.

The relevant Maxwell equations are

$$
\dot{B}_{z}=-(\nabla \times E)_{z}, \quad \nabla \times \mathbf{B}=\mu_{0} \mathbf{J} \delta(z), \quad \nabla \cdot \mathbf{B}=0,
$$

with $\nabla \cdot \mathbf{J}=0$. Together with the material law, Eq. (1), the electrodynamics can be written as a nonlocal and nonlinear diffusion equation. This equation is solved by an efficient Fourier real-space hybrid algorithm with boundary conditions assuring $J=0$ in the antidots, as explained in [37]. The parameters chosen for the simulations are $T_{c}=9.2 \mathrm{~K}, j_{c 0}=$ $j_{c 0} / d=1.2 \times 10^{11} \mathrm{~A} / \mathrm{m}^{2}, \rho_{0}=6 \times 10^{-9} \Omega \mathrm{m}$, and $T_{0}=$ $0.4 T_{c}$. The thermal parameters $K, h$, and $c$ follow cubic temperature dependencies with $K\left(T=T_{c}\right)=20 \mathrm{~W} / \mathrm{Km}$ and $c\left(T=T_{c}\right)=2 \times 10^{4} \mathrm{~J} / \mathrm{Km}^{3}$. Other values indicated below also correspond to the parameters at $T=T_{c}$.

The simulation was conducted in two steps. First, the magnetic field was slowly ramped up with thermal feedback turned off, i.e., with no avalanches and at isothermal conditions everywhere. Second, the thermal feedback was turned on and the avalanche was nucleated by a heat pulse at the edge. This procedure is described in [37]. All main features of the avalanches occurring on the samples studied here can be properly reproduced by simulations conducted using this strategy. Figure 4 shows the time evolution of the magnetic field, $B_{z}$; the current streamlines, $J$; the temperature, $T$; and the electric field, $E$, for a sample in the form of a strip of width $2 w$ and thickness $d \ll w$, consisting of a superconducting film patterned with a square lattice of square antidots. The side of the ADs is $0.02 w$ and the lattice parameter is $0.06 w$ with $w=2 \mathrm{~mm}$. The total area, including vacuum all around the stripe, needed for boundary conditions is discretized on

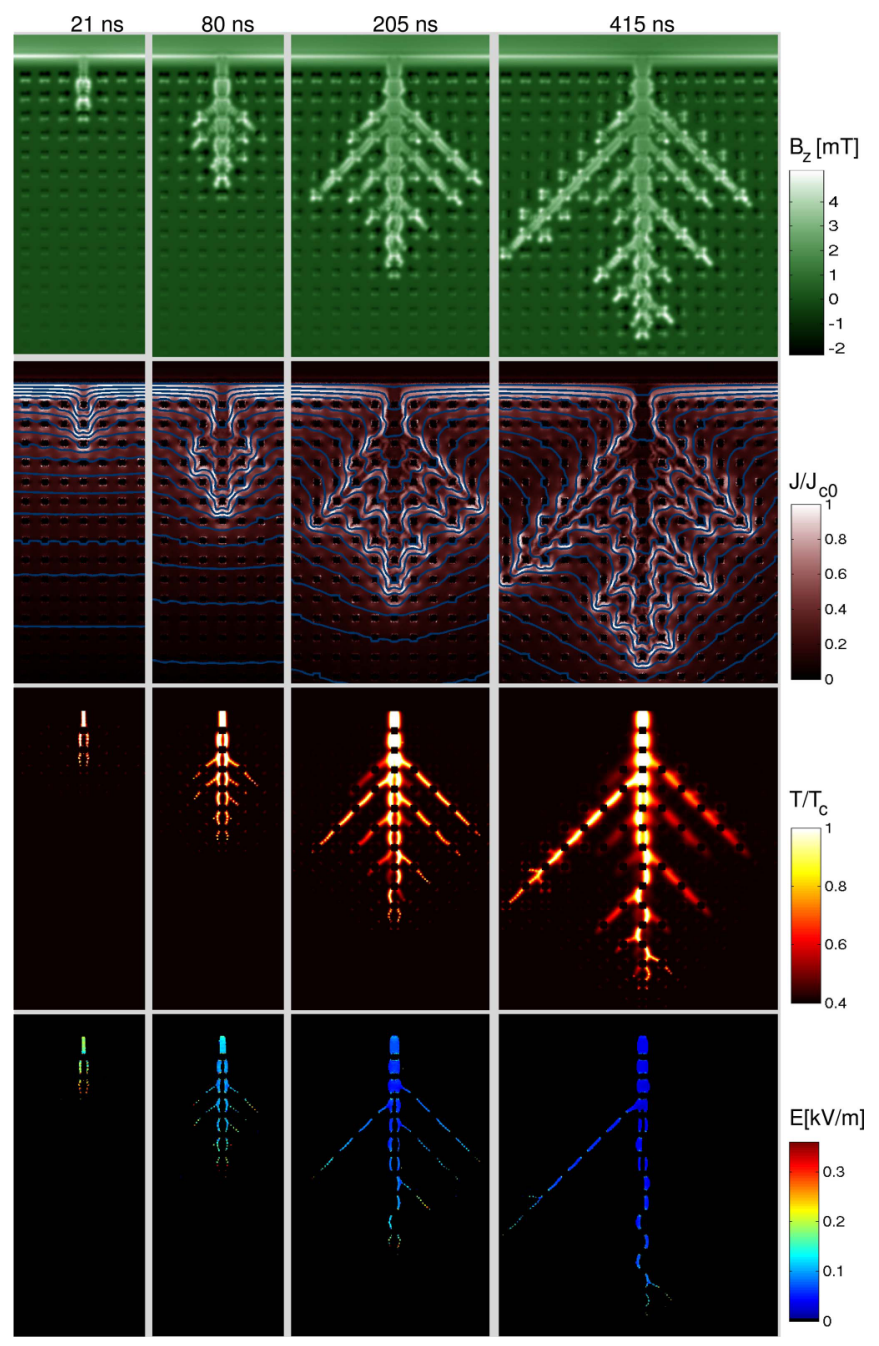

FIG. 4. (Color online) Flux avalanche in a square array of square antidots, reproducing avalanches with the Christmas Tree morphology (see text) occurring for $T / T_{c}=0.4$ and $H=5.3$ Oe with the typical parameters of a $\mathrm{Nb}$ thin film. The first set of panels represents the distribution of the magnetic-flux density $B_{z}$, the second set shows the induced sheet current $J$, the third presents the map of the reduced temperature, and the last depicts the space distribution of the electric field. Time evolves from left to right: $21,80,205$, and 415 ns.

a $1536 \times 1024$ equidistant grid. In addition, $h\left(T=T_{c}\right)=$ $2 \times 10^{3} \mathrm{~W} / \mathrm{Km}^{2}, n=90 T_{c} / T-50$, and $d=100 \mathrm{~nm}$. The panels depict, for an avalanche triggered by an applied field $H=5.3 \mathrm{Oe}$, the space distribution of the above-mentioned physical quantities, from its early stage at instant $t=21 \mathrm{~ns}$, to an almost fully developed treelike pattern, at $t=415 \mathrm{~ns}$.

The first horizontal row of panels in Fig. 4 shows maps of the magnetic-field distribution, from which one can follow the formation of the Christmas Tree morphology, in full accordance with the experimental results for square lattices of square antidots: the main trunk is perpendicular to the sample edge, running along one of the main directions of the lattice of ADs. Notice that the branching of the simulated avalanche is not perfectly symmetric on both sides. This effect arises from the fact that the nucleation point is not aligned with the antidot array and therefore the avalanche is asymmetric from 
the very beginning. The second set of panels is a map of the current density, evidencing that its largest values (light colors) occur at the AD tips; current streamlines appear as if they were delineating the trunk and branches of the flux avalanche. The third set of snapshots depicts the local temperature, confirming that the heat developed by vortex motion can elevate $T$ to values as high as $T_{c}$, further facilitating flux invasion into the film. The last set contains maps of the local electric field: at each stage one can anticipate which branches are likely to develop and grow, simply by monitoring the spots where $E$ is more intense. Interestingly, the evolution of thermomagnetic avalanches exhibits some fundamental differences with respect to their behavior in a plain superconducting sample without antidots [34]. Indeed, for a sample with antidots, the main trunk propagation is locally not strictly straight, since the branches propagate from the corners of the antidots. This gives some peculiar S-like patterns in the main trunk of $E$ and $T$ although they are not not visible in $B_{z}$. Notice that the heat does not propagate through the ADs, which means that the thermomagnetic avalanches propagate through the ADs driven only by their magnetic component. In a sense, the avalanches stop at each AD and are nucleated anew at the corners, following a repetitive start-stop propagation.

It is worth noticing that, since avalanches develop so fast that they cannot be recorded while in progress, being able to obtain reliable information from simulations can be of great value. Such is the case of the local values of temperature and electric field, as described above, most especially because measuring locally such quantities would be nearly unfeasible in practice.

\section{Lattice symmetry}

A further example of how symmetry and geometry combine to form the avalanche morphology is given in Fig. 5, where simulated [38] (a) and measured (b) results for a sample decorated with a centered rectangular 2D Bravais lattice of square ADs are compared. The MO images are for sample MoGe-III at $H=1.0 \mathrm{Oe}$ and the simulations were performed for a specimen designed to match its features. Both panels show that flux avalanches follow the characteristic angles of the structure, $\alpha$ and $\theta=180^{\circ}-2 \alpha$. Using 34 avalanches recorded at a variety of values of the temperature and the applied magnetic field, we have estimated the average values of these angles: $\alpha=(62.0 \pm 2.0)^{\circ}$ (averaged over 20 branches) and $\theta=(54.0 \pm 1.3)^{\circ}(14$ branches $)$. Both values are comparable, within experimental error, with the nominal values of 63.4 and $53.2^{\circ}$, respectively. Noticeably, avalanches occurring at the bottom and top edges exhibit a main trunk which, however, is absent on the side edges. This feature is a direct consequence of the existence, in the lattice, of straight paths connecting ADs in the direction orthogonal to the upper and lower edges, but not to the lateral ones.

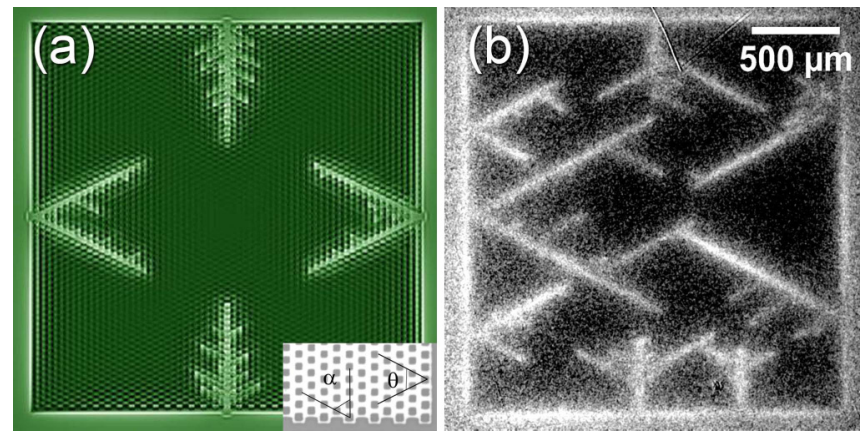

FIG. 5. (Color online) (a) Magnetic-flux distribution for $H=$ 3.2 Oe, obtained from simulations for a superconducting film decorated with a centered rectangular 2D Bravais lattice of square ADs, designed to match sample MoGe-III. (b) MO image taken at $3 \mathrm{~K}$ and $1.0 \mathrm{Oe}$ for sample MoGe-III. Inset: Optical image showing the nominal angles $\alpha=63.4^{\circ}$ and $\theta=53.2^{\circ}$ of the lattice.

\section{CONCLUSIONS}

In summing up, we have employed MO imaging to visualize the occurrence of flux avalanches in superconducting films of polycrystalline $\mathrm{Nb}$ and amorphous $\mathrm{Mo}_{79} \mathrm{Ge}_{21}$. For specimens with square antidots arranged in a square lattice, avalanches have the form of Christmas Trees, for which the main trunk is perpendicular to the sample edge and the branches develop at an angle of $45 \mathrm{deg}$ with the main axes of the antidot lattice. The overall features of the avalanches, and in particular the 45-deg direction of the branches, were confirmed by numerical simulations using the thermomagnetic model. The habit of growing like a tree with inclined branches can be explained as a consequence that concave corners are preferential spots for flux penetration, regardless of the sample size being in the microscopic or macroscopic regimes. By choosing to study films of $\mathrm{Nb}$ and amorphous $\mathrm{Mo}_{79} \mathrm{Ge}_{21}$, we were able to conclude that, although strongly dependent on the lattice symmetry and antidot geometry, the avalanche morphology is not sensitive to $\kappa$.

\section{ACKNOWLEDGMENTS}

This work was partially supported by the Fonds de la Recherche Scientifique (F.R.S.-FNRS), the Methusalem Funding of the Flemish Government, the Fund for Scientific Research-Flanders (FWO-Vlaanderen), the Brazilian National Council for Scientific and Technological Development (CNPq) and the São Paulo Research Foundation (FAPESP) Grant No. 2007/08072-0, and the program for scientific cooperation F.R.S.-FNRS-CNPq. J. V.d.V. acknowledges support from FWO-Vlaanderen. The work of A.V.S. is partially supported by "Mandat d'Impulsion Scientifique" of the F.R.S.FNRS and by the Fonds spéciaux pour la Recherche - Crédit de démarrage, University of Liège.
[1] U. Bolz, B. Biehler, D. Schmidt, B.-U. Runge, and P. Leiderer, Europhys. Lett. 64, 517 (2003).

[2] L. Niemeyer, L. Pietronero, and H. J. Wiesmann, Phys. Rev. Lett. 52, 1033 (1984).
[3] L. Fernandez and F. Guinea, J. Phys. A 21, L301 (1988).

[4] V. A. Rakov and M. A. Uman, Lightning: Physics and Effects (Cambridge University Press, Cambridge, 2003). 
[5] R. G. Mints and A. L. Rakhmanov, Rev. Mod. Phys. 53, 551 (1981).

[6] R. G. Mints and E. H. Brandt, Phys. Rev. B 54, 12421 (1996).

[7] I. S. Aranson, A. Gurevich, M. S. Welling, R. J. Wijngaarden, V. K. Vlasko-Vlasov, V. M. Vinokur, and U. Welp, Phys. Rev. Lett. 94, 037002 (2005).

[8] D. Y. Vodolazov and F. M. Peeters, Phys. Rev. B 72, 172508 (2005).

[9] J. Gutierrez, B. Raes, J. Van de Vondel, A. V. Silhanek, R. B. G. Kramer, G. W. Ataklti, and V. V. Moshchalkov, Phys. Rev. B 88, 184504 (2013).

[10] A. Gurevich and M. Friesen, Phys. Rev. B 62, 4004 (2000).

[11] D. Cerbu, V. N. Gladilin, J. Cuppens, J. Fritzsche, J. Tempere, J. T. Devreese, V. V. Moshchalkov, A. V. Silhanek, and J. Van de Vondel, New J. Phys. 15, 063022 (2013).

[12] J. R. Clem and K. K. Berggren, Phys. Rev. B 84, 174510 (2011).

[13] G. Via, C. Navau, and A. Sanchez, J. Appl. Phys. 113, 093905 (2013).

[14] O.-A. Adami et al., Appl. Phys. Lett. 102, 052603 (2013).

[15] J. I. Vestgården and T. H. Johansen, Supercond. Sci. Technol. 25, 104001 (2012).

[16] A. L. Rakhmanov, D. V. Shantsev, Y. M. Galperin, and T. H. Johansen, Phys. Rev. B 70, 224502 (2004).

[17] D. V. Denisov, A. L. Rakhmanov, D. V. Shantsev, Y. M. Galperin, and T. H. Johansen, Phys. Rev. B 73, 014512 (2006).

[18] V. Vlasko-Vlasov, U. Welp, V. Metlushko, and G. W. Crabtree, Physica C 341-348, 1281 (2000).

[19] M. Menghini, R. J. Wijngaarden, A. V. Silhanek, S. Raedts, and V. V. Moshchalkov, Phys. Rev. B 71, 104506 (2005).

[20] M. Motta, F. Colauto, R. Zadorosny, T. H. Johansen, R. B. Dinner, M. G. Blamire, G. W. Ataklti, V. V. Moshchalkov, A. V. Silhanek, and W. A. Ortiz, Phys. Rev. B 84, 214529 (2011).

[21] I. Aranson, A. Gurevich, and V. Vinokur, Phys. Rev. Lett. 87, 067003 (2001).

[22] M. S. Welling, R. J. Wijngaarden, C. M. Aegerter, R. Wördenweber, and P. Lahl, Physica C 404, 410 (2004).
[23] T. Tamegai, Y. Tsuchiya, Y. Nakajima, T. Yamamoto, Y. Nakamura, J. S. Tsai, M. Hidaka, H. Terai, and Z. Wang, Physica C 470, 734 (2010).

[24] N. Nakai and M. Machida, Physica C 470, 1148 (2010).

[25] P. de Gennes, Superconductivity of Metals and Alloys (Westview, Boulder, 1999).

[26] P. H. Kes and C. C. Tsuei, Phys. Rev. B 28, 5126 (1983).

[27] C. Jooss, J. Albrecht, H. Kuhn, S. Leonhardt, and H. Kronmüller, Rep. Prog. Phys. 65, 651 (2002).

[28] L. E. Helseth, R. W. Hansen, E. I. Ilyashenko, M. Baziljevich, and T. H. Johansen, Phys. Rev. B 64, 174406 (2001).

[29] T. Schuster, H. Kuhn, E. H. Brandt, M. V. Indenbom, M. Klaser, G. Muller-Vogt, H.U. Habermeier, H. Kronmuller, and A. Forkl, Phys. Rev. B 52, 10375 (1995).

[30] M. Pannetier, R. J. Wijngaarden, I. Floan, J. Rector, B. Dam, R. Griessen, P. Lahl, and R. Wördenweber, Phys. Rev. B 67, 212501 (2003).

[31] A. V. Silhanek, M. V. Milosević, R. B. G. Kramer, G. R. Berdiyorov, J. Van de Vondel, R. F. Luccas, T. Puig, F. M. Peeters, and V. V. Moshchalkov, Phys. Rev. Lett. 104, 017001 (2010).

[32] Th. Schuster, H. Kuhn, and E. H. Brandt, Phys. Rev. B 54, 3514 (1996).

[33] Notice that, unlike for sample Nb-I, $45^{\circ}$ avalanches are clearly visible in the $\mathrm{Nb}$-II sample after zero-field cooling. This is likely a consequence of a weaker disorder in sample Nb-II.

[34] J. I. Vestgården, D. V. Shantsev, Y. M. Galperin, and T. H. Johansen, Sci. Rep. 2, 886 (2012).

[35] P. Mikheenko, A. J. Qviller, J. I. Vestgården, S. Chaudhuri, I. J. Maasilta, Y. M. Galperin, and T. H. Johansen, Appl. Phys. Lett. 102, 022601 (2013).

[36] D. V. Denisov, A. L. Rakhmanov, D. V. Shantsev, Y. M. Galperin, and T. H. Johansen, Phys. Rev. B 73, 014512 (2006).

[37] J. I. Vestgården, P. Mikheenko, Y. M. Galperin, and T. H. Johansen, New J. Phys. 15, 093001 (2013).

[38] For this particular simulation the parameters are $h\left(T=T_{c}\right)=$ $2 \times 10^{2} \mathrm{~W} / \mathrm{K} \mathrm{m}^{2}, n=50 T_{c} / T, w=2.5 \mathrm{~mm}, d=60 \mathrm{~nm}$, grid size $1024 \times 1024$, AD width $0.015 w$, AD center-to-center distance $0.03 w$. 